\title{
NEUROFIBROMATOSE TIPO 1 SEGMENTAR OU EM MOSAICO: A PROPÓSITO DE 2 DOENTES
}

\author{
Paulo Morais', Fortunato Vieira² \\ 'Assistente Hospitalar de Dermatologia e Venereologia/Consultant of Dermatology, Serviço de Dermatologia e \\ Venereologia, Centro Hospitalar Tondela-Viseu EPE, Viseu, Portugal \\ ${ }^{2}$ Assistente Hospitalar de Anatomia Patológica/Consultant of Pathology, Histocit - Laboratório de Anatomia Patológica, \\ Trofa, Portugal
}

RESUMO - A neurofibromatose tipo 1 (NF1) segmentar caracteriza-se pela presença das manifestações típicas da NF1 circunscritas a um ou mais segmentos corporais e geralmente localizadas unilateralmente. É causada por uma mutação pós-zigótica somática, e mais raramente gonadal, no gene NF1, sendo por isso um exemplo de mosaicismo genético e não uma forma distinta de neurofibromatose. Apresentamos dois doentes com NF1 segmentar e efectuamos uma revisão actualizada do tema.

PALAVRAS-CHAVE - Genes da neurofibromatose 1; Mosaicismo; Neurofibromatose 1.

\section{SEGMENTAL OR MOSAIC NEUROFIBROMATOSIS TYPE 1: A REPORT OF 2 PATIENTS}

ABSTRACT - Segmental neurofibromatosis type 1 (NF1) is characterized by typical features of NF1 limited to one or more body segments, and usually located unilaterally. It is caused by a somatic (rarely gonadal) postzygotic mutation in the NF1 gene, explaining why it should be regarded as a genetic mosaicism instead of a distinct entity from neurofibromatosis. We herein report two patients with segmental NF1 and give an updated overview of the disease.

KEY-WORDS - Genes, Neurofibromatosis 1; Mosaicism.

\footnotetext{
Conflitos de interesse: Os autores declaram não possuir conflitos de interesse.

No conflicts of interest.

Suporte financeiro: $O$ presente trabalho não foi suportado por nenhum subsídio ou bolsa.

No sponsorship or scholarship granted.

Direito à privacidade e consentimento escrito / Privacy policy and informed consent: Os autores declaram que pediram consentimento ao doente para usar as imagens no artigo. The authors declare that the patient gave written informed consent for the use of its photos in this article.
}

Recebido/Received - Dezembro/December 2013; Aceite/Accepted - Janeiro/January 2014

Por decisão dos autores, este artigo não foi redigido de acordo com os termos do novo Acordo Ortográfico.

\section{Correspondência:}

\section{Dr. Paulo Morais}

Serviço de Dermatologia e Venereologia

Centro Hospitalar Tondela-Viseu, EPE

Av. Rei D. Duarte

3509-504 Viseu

Portugal

E-mail: paulomoraiscardoso@gmail.com 


\section{Caso Clínico}

\section{INTRODUÇÃO}

As neurofibromatoses (NF) são entidades clinica e geneticamene heterogéneas incluídas no grupo das síndromes neurocutâneas (facomatoses). Adicionalmente, a NF tipo 1 (NF1), ou doença de von Recklinghausen, pertence ao grupo das RASopatias', sendo a forma de NF mais frequente. A NF tipo 5 (NF5) ou segmentar, é uma doença rara, caracterizada, segundo $\operatorname{Riccardi}^{2}$ (Tabela 1), por manifestações da NFI circunscritas a um segmento corporal. Apresentamos dois doentes com expressão segmentar da NF1 e efectuamos uma breve revisão da literatura referente ao tema.

\section{CASOS CLÍNICOS}

Uma mulher de 57 anos de idade (Doente 1), com história de dislipidemia e medicada com sinvastatina $20 \mathrm{mg} /$ dia, foi orientada para a consulta de Dermatologia pela presença de nódulos ocasionalmente dolorosos, com crescimento progressivo e mais de 30 anos de evolução, localizados no tronco. Ao exame objectivo observámos várias lesões pápulo-nodulares com 5-15 mm de diâmetro, eritemato-rosadas, moles, deprimíveis, bem delimitadas, agrupadas e localizadas na região infraclavicular esquerda (Fig.s 1A e 1B). O restante exame físico era normal. A história familiar não revelou parentes acometidos, sendo o único filho da doente saudável. A tomografia computorizada (TC) cerebral, a ecografia abdominal e a avaliação oftalmológica não evidenciaram alterações relevantes. Efectuou-se a excisão de uma das lesões. $\bigcirc$ exame histopatológico mostrou a presença de um nódulo de crescimento expansivo na derme reticular superficial e profunda, constituído por células fusiformes e alguns mastócitos entremeados em tecido fibroso, sugestivo de neurofibroma cutâneo (Fig.s 1C e 1D). Os achados clínicos e histológicos eram compatíveis com o diagnóstico de NF1 segmentar. Explicada a natureza e a evolução expectável das lesões optou-se pela abstenção terapêutica.

Um homem de 64 anos (Doente 2), saudável, foi observado na nossa consulta por lesões assintomáticas com características similares às da doente 1, mas em menor número, localizadas na face anterolateral do braço esquerdo, em disposição grosseiramente linear (Fig.s 2A e 2B). O quadro apresentava cerca de 25 anos de evolução. O restante exame físico e a avaliação oftalmológica eram normais. A história familiar era negativa, sendo os descendentes do doente saudáveis. A TC cerebral e a ecogafia abdominal não mostraram alterações de relevo. Foi efectuada biópsia excisional de uma das lesões. O exame histológico foi compatível com o diagnóstico de neurofibroma cutâneo (Fig. 2C). Tendo em conta o quadro clínico e o exame anátomo- patológico foi estabelecido o diagnóstico de NF1 segmentar. Por desejo do doente foi efectuado tratamento cirúrgico das lesões com laser $\mathrm{CO}_{2}$.

\section{DISCUSSÃO}

Actualmente, as neurofibromatoses classificam-se em NF tipo 1 (NF1), NF tipo 2 (NF2), e outras variantes

Tabela 1 - Classificação das neurofibromatoses segundo Riccardi².

Clássica ou doença de von Recklinghausen (NF1)

Acústica (NF2)

Mista (NF3)

Variante (NF4)

Segmentar (NF5)

MCCL familiares (NF6)

Início tardio (NF7)

Não Especificada (NF8)

\section{CARACTERÍSTICAS}

$90 \%$ dos casos, MCCL, neurofibromas, nódulos de Lisch, efélides axilares, alterações ósseas e neurológicas, neoplasias benignas e malignas

Neuromas do acústico bilaterais, poucas $\mathrm{MCCL}$ e neurofibromas

Combina características da NF1 e NF2

Variações nas MCCL, neurofibromas, neoplasias do SNC, nódulos de Lisch

MCCL e/ou neurofibromas com distribuição segmentar

Múltiplas MCCL, sem neurofibromas

Início após os 20 anos, neurofibromas, poucas MCCL

Aspectos variáveis

$\mathrm{MCCL}$ - manchas café-com-leite, NF - neurofibromatose, SNC - sistema nervoso central. 


\section{Caso Clínico}

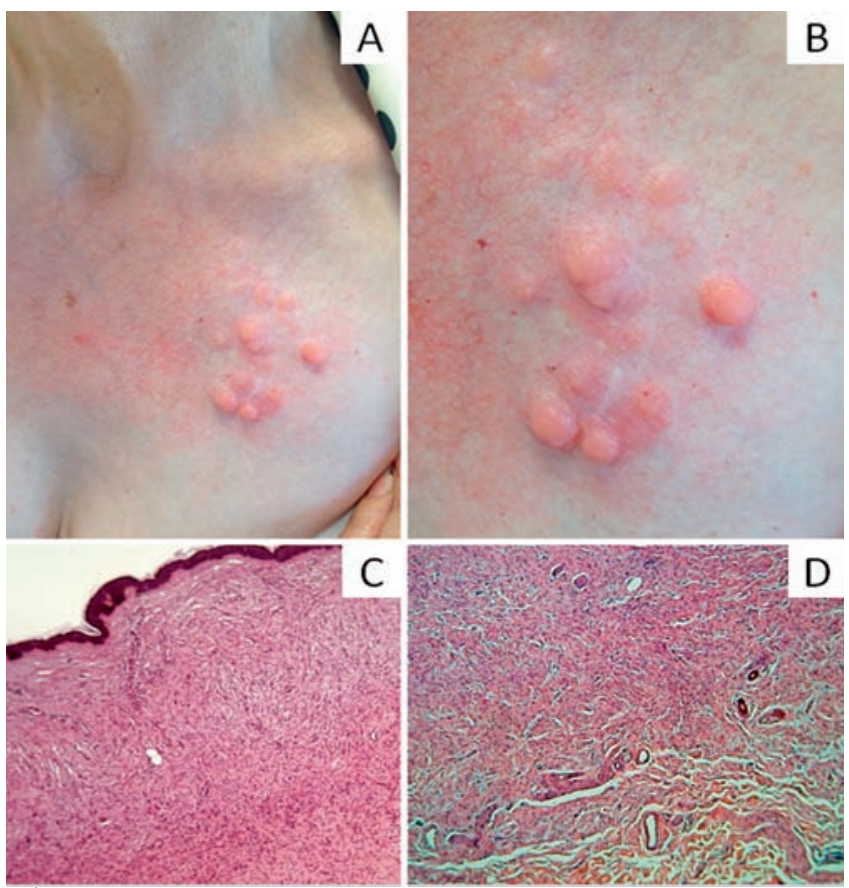

Fig 1 - Aspecto clínico da doente 1 (A); detalhe das lesões (B). Exame histológico $(H \& E, 5 x)$ de lesão excisada mostrando uma proliferação dérmica constituída por células fusiformes e alguns mastócitos entremeados em tecido fibroso, na ausência de pleomorfismo nuclear, mitoses ou necrose, aspectos sugestivos de neurofibroma cutâneo (C, D).

atípicas ou entidades relacionadas, incluindo a schwannomatose, as formas segmentares de NF1, NF2 e schwannomatose, as manchas café-com leite (MCCL) familiares e a NF1 fenótipo Noonan (síndrome Noonan/NF1), entre outras ${ }^{3-5}$. A NF1 segmentar e a NF2 segmentar não devem ser interpretadas como entidades distintas da NF1 e NF2, respectivamente, mas como formas localizadas ou em mosaico 6 . A Tabela $2^{6-13}$ resume a evolução da designação "NF segmentar".

A NF1 segmentar é cerca de 10-20 vezes menos frequente do que a NF1, apresentando uma prevalência estimada de 0,0027\% (1 em cada 36000-40000 indivíduos) $)^{6,14}$. É, por isso, mais frequente do que a NF2. Afecta primariamente caucasianos, atingindo duas vezes mais mulheres do que homens. A idade de início é bimodal, com picos entre os 10-30 anos e entre os 50-70 anos ${ }^{15-17}$. Caracteriza-se pela presença de lesões pigmentares (MCCL e/ou efélides intertriginosas) e/ou neurofibromas (cutâneos, subcutâneos ou plexiformes) geralmente com distribuição segmentar e unilateral, não cruzando a linha média, na ausência de história familiar da doença e de envolvimento

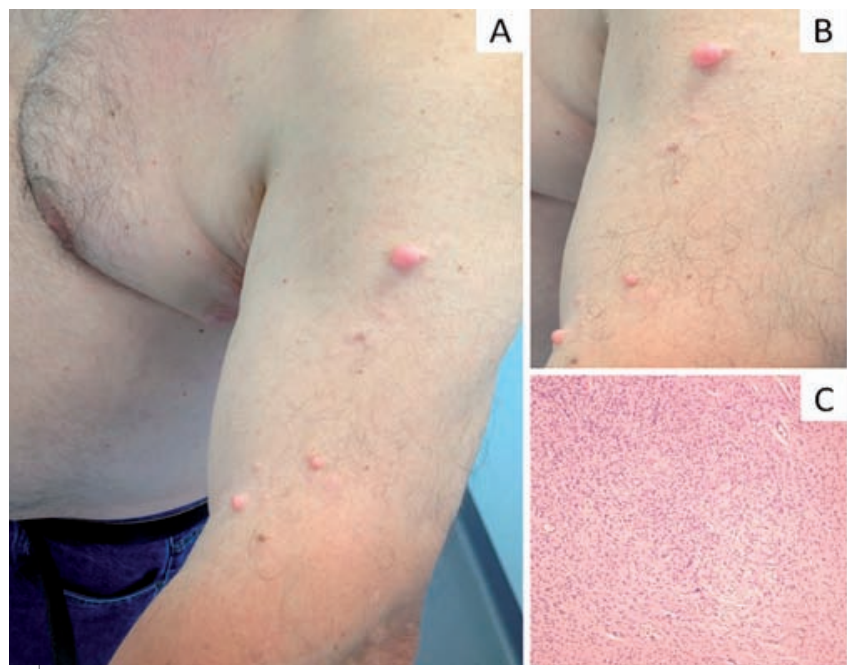

Fig 2 - Aspecto clínico do doente 2 (A); detalhe das lesões (B). Exame histológico (H\&E, 10x) da peça excisada mostando aspectos compatíveis com neurofibroma cutâneo.

sistémico $^{2,18}$. No entanto, menos frequentemente, podem estar envolvidos mais do que um segmento corporal, uni ou bilateralmente (6\%), tanto de forma simétrica como assimétrica ${ }^{6,13,19-22}$, reflectindo os padrões de mosaicismo propostos por Happle ${ }^{23}$. A apresentação mais habitual é a de neurofibromas cutâneos localizados, tal como observado nos nossos doentes. As lesões podem ter uma apresentação focal ou zosteriforme seguindo um dermátomo. A área mais frequentemente afectada é a cervical, seguida pelas regiões torácica, lombar e sagrada ${ }^{22}$. O atingimento de um membro, conforme constatado no doente 2, é uma forma de apresentação rara $^{24}$. Nos doentes com alterações da pigmentação as lesões tendem a seguir as linhas de Blaschko unilateralmente ${ }^{22}$. Contrariamente ao definido por Riccardi ${ }^{2}$, sabe-se actualmente que, apesar de extremamente raro, pode ocorrer envolvimento sistémico e transformação maligna na NF segmentar, incluindo: dificuldades de aprendizagem, nódulos de Lisch, gliomas da via óptica, pseudoartrose, displasia da asa do esfenóide, NF visceral, agenesia renal unilateral, tumores malignos da bainha dos nervos periféricos e carcinoma gástrico ${ }^{18,25-28}$. Para serem compatíveis com o diagnóstico de NF1 segmentar os nódulos de Lisch devem ser unilaterais e ipsilaterais ao dermátomo envolvido. No caso de serem bilaterais sugerem o diagnóstico de $\mathrm{NF}^{26}$. Clinicamente, as manifestações da doença apresentam um curso similar às da NF1 generalizada, com as alterações da pigmentação e os neurofibromas plexiformes a desenvolverem-se na 


\section{Caso Clínico}

Tabela 2 - Evolução da definição de neurofibromatose segmentar ${ }^{6-13}$.

\begin{tabular}{|c|c|}
\hline $\begin{array}{l}\text { Gammel }(1931)^{7} \\
\text { Crowe et al. }(1956)^{8}\end{array}$ & $\begin{array}{l}\text { Primeiras descrições de NF segmentar. } \\
\text { Utilização da designação neurofibromatose sectorial. }\end{array}$ \\
\hline Miller e Sparkes (1977) ${ }^{9}$ & Primeira utilização do termo NF segmentar \\
\hline Riccardi $(1982)^{2}$ & $\begin{array}{l}\text { Classificação da NF em } 8 \text { subtipos (NF1 a NF8, ou NF-I a NF-VIII). } \\
\text { Definição da NF5 ou segmentar: MCCL e/ou neurofibromas em distribuição segmentar } \\
\text { e unilateral, sem cruzar a linha média, ausência de história familiar de NF e de } \\
\text { envolvimento sistémico. }\end{array}$ \\
\hline Roth et al. $(1987)^{10}$ & $\begin{array}{l}\text { Subclassificação da NF segmentar em } 4 \text { categorias: segmentar verdadeira (tipo } 5 \text { de } \\
\text { Riccardi), localizada com envolvimento profundo, hereditária e bilateral. }\end{array}$ \\
\hline Tinschert et al. $(2000)^{11}$ & $\begin{array}{l}\text { A NF segmentar é causada por mutações somáticas no gene NFl, razão pela qual não } \\
\text { deve ser interpretada como uma entidade distinta da NFl. }\end{array}$ \\
\hline Ruggieri e Hudson (2001) ${ }^{13}$ & $\begin{array}{l}\text { Divisão das manifestações clínicas de NF1 segmentar em } 4 \text { grupos: apenas alterações da } \\
\text { pigmentação, apenas neurofibromas, combinação de ambos os aspectos, neurofibromas } \\
\text { plexiformes isolados. } \\
\text { Aplicação dos termos NF1 "em mosaico localizada" e NF1 "em mosaico generalizada" } \\
\text { para descrever a NF segmentar e a NF1 generalizada, respectivamente, de forma a } \\
\text { reflectir a patogénese da doença. }\end{array}$ \\
\hline Ruggieri $(2001)^{6}$ & $\begin{array}{l}\text { Tanto a NF1, como a NF2 e a schwannomatose podem apresentar manifestações } \\
\text { segmentares. Sugere-se a adopção dos termos NF1 segmentar (em mosaico), NF2 } \\
\text { segmentar (em mosaico) e schwannomatose segmentar (em mosaico) e o abandono da } \\
\text { designação NF tipo } 5 \text {. }\end{array}$ \\
\hline
\end{tabular}

infância e os neurofibromas na vida adulta ${ }^{21}$.

O diagnóstico diferencial da NF1 segmentar é amplo e inclui tumores benignos (tricoepitelioma, leiomiomas), tumores malignos (carcinomas, linfomas), nevo epidérmico, nevo lipomatoso cutâneo superficial, lentiginose agminada, sarcoidose, granuloma anular e xantomas ${ }^{22}$.

A NF1 segmentar resulta de uma mutação somática durante a embriogénese (pós-zigótica) no gene NF1, localizado no braço longo do cromossoma 17, sendo por isso um exemplo claro de mosaicismo genético ${ }^{11,13}$. Se a mutação somática ocorrer precocemente, antes da diferenciação tecidular, resulta em doença generalizada (NF1 ou "em mosaico generalizada"). No caso de ocorrer mais tardiamente afecta apenas uma linhagem celular, limitando as alterações a uma região ou órgão (NF1 segmentar ou "em mosaico localizada") $)^{13}$. A mutação pode também ocorrer em células da linhagem gonadal, o que poderá justificar os casos de doentes com doença localizada que geram descendentes com NF1 generalizada ou, menos frequentemente, NF1 segmentar ${ }^{13,29,30}$.
A NF1 segmentar encontra-se provavelmente subdiagnosticada uma vez que a maioria dos doentes afectados são assintomáticos e não valorizam a doença, procurando cuidados médicos apenas por razões estéticas. Não é raro o diagnóstico acidental aquando do exame físico por motivo distinto.

Embora não existam directrizes específicas relativas à abordagem dos doentes com NF segmentar, recomenda-se um seguimento cuidadoso, especialmente de doentes jovens, no sentido de monitorizar a progressão da doença ou detectar qualquer complicação sistémica que possa ocorrer. Os doentes devem ser submetidos a um exame físico detalhado no sentido de procurar neurofibromas e MCCL noutros locais e avaliar a doença ocular ${ }^{16}$. Na verdade, mesmo que o doente se inclua inicialmente na definição de NF segmentar, com o tempo pode desenvolver lesões dispersas ou nódulos de Lisch bilaterais e a doença terá de ser reclassificada, o que justifica a avaliação regular destes doentes ${ }^{16}$. Os doentes devem ser informados de que não sofrem de NF1 generalizada e do risco reduzido de complicações associadas. Doentes que planeiem 


\section{Caso Clínico}

ter filhos devem alertados para o pequeno risco de transmissão da doença à descendência. Dados extrapolados de estudos em animais sugerem que este risco é proporcional à percentagem da superfície corporal atingida $^{13}$.

\section{REFERÊNCIAS}

1. Raven KA. The RASopathies. Annu Rev Genomics Hum Genet. 2013; 14:355-69.

2. Riccardi VM. Neurofibromatosis: clinical heterogeneity. Curr Probl Cancer. 1982; 8:1-34.

3. Carey JC, Baty BJ, Johnson JP, Morrison T, Skolnik $M$, Kivlin J. The genetic aspects of neurofibromatosis. Ann NY Acad Sci. 1986; 486:45-6.

4. Neurofibromatosis. Conference statement. National Institutes of Health Consensus Development Conference. Arch Neurol. 1988; 45:575-8.

5. Chen SL, Liu C, Liu B, Yi CJ, Wang ZX, Rong YB, et al. Scwannomatosis: A new member of neurofibromatosis family. Chin Med J. 2013; 126:2656-60.

6. Ruggieri M. Mosaic (segmental) neurofibromatosis type 1 (NF1) and type 2 (NF2): no longer neurofibromatosis type 5 (NF5). Am J Med Genet. 2001; 101:178-80.

7. Gammel JA. Localized neurofibromatosis. Arch Dermatol Syph. 1931; 24:712-5.

8. Crowe FW, Schull WJ, Neel JV. Clinical, pathological, and genetic study of multiple neurofibromatosis. Springfield: Charles C Thomas; 1956.

9. Miller RM, Sparkes RS. Segmental neurofibromatosis. Arch Dermatol. 1977; 123:837-8.

10. Roth RR, Martines R, James WD. Segmental neurofibromatosis. Arch Dermatol. 1987; 123:91720.

11. Tinschert S, Naumann I, Stegmann E, Buske A, Kaufmann D, Thiel G, et al. Segmental neurofibromatosis is caused by somatic mutation of the neurofibromatosis type 1 (NF1) gene. Eur J Hum Genet. 2000; 8:455-9.

12. Weiss SW, Goldblum JR. Enzinger and Weiss's Soft Tissue Tumors. 4th ed. St Louis: Mosby; 2001.

13. Ruggieri M, Huson SM. The clinical and diagnostic implications of mosaicism in the neurofibromatoses. Neurology. 2001; 56:1433-43.

14. MacCollin M, Jacoby LB, Jones D, Ojemann R, Feit $H$, Gusella J. Somatic mosaicism of the neurofibromatosis type 2 suppressor-gene. Neurology. 1997; 48:A429.

15. Ingordo V, D'Andria G, Mendicini S, Grecucci M,
Baglivo A. Segmental neurofibromatosis: is it uncommon or underdiagnosed? Arch Dermatol. 1995; 131:959-60.

16. Gabhane SK, Kotwal MN, Bobhate S. Segmental neurofibromatosis: a report of 3 cases. Indian J Dermatol. 2010; 55:105-8.

17. Niiyama S, Satoh K, Kaneko S, Aiba S, Mukai H. Segmental neurofibromatosis. Acta Derm Venereol. 2005; 85:448-9.

18. Listernick R, Mancini AJ, Charrow J. Segmental neurofibromatosis in childhood. Am J Med Genet. 2003; 121A:132-5.

19. Nagaoka Y, Asahina A, Yano S, Tamaki K. Bilateral segmental neurofibromatosis. Acta Derm Venereol. 2002; 82:219-20.

20. Gonzalez G, Russi ME, Lodeiros A. Bilateral segmental neurofibromatosis: a case report and review. Pediatr Neurol. 2007; 36:51-3.

21. Hudson SM, Ruggieri M. The neurofibromatosis. In: Harper J, Oranje JM, Rose M, editors. Textbook of Pediatric Dermatology. Vol. 2. Oxford: Blackwell Science Publishers; 2000.p. 1204-24.

22. Hager CM, Cohen PR, Tschen JA. Segmental neurofibromatosis: case reports and review. J Am Acad Dermatol. 1997; 37:864-9.

23. Happle R. Pigmentary patterns associated with human mosaicism: a proposed classification. Eur J Dermatol. 1993; 3:170-4.

24. Ilyas AM, Nourissat G, Jupiter JB. Segmental neurofibromatosis of the hand and upper extremity: a case report. J Hand Surg Am. 2007; 32:1538-42.

25. Victor FC. Segmental neurofibromatosis. Dermatol Online J. 2005; 11:20-1.

26. Nicita F, lannetti L, Spalice A, Papetti L, Ursitti F, Properzi $E$, et al. Unilateral Lisch nodules in a 47-year-old woman without other stigmata of neurofibromatosis type I: An example of segmental neurofibromatosis? Ophthalmic Genet. 2013; 34:178-9.

27. Kajimoto A, Oiso N, Fukai K, Ishii M. Bilateral segmental neurofibromatosis with gastric carcinoma. Clin Exp Dermatol. 2007; 32:43-4.

28. Schwarz J, Belzberg AJ. Malignant peripheral nerve sheath tumors in the setting of segmental neurofibromatosis. Case report. J Neurosurg. 2000; 92:342-6.

29. Moss C, Green SH. What is segmental neurofibromatosis? Br J Dermatol. 1994; 130:106-10.

30. Oguzkan S, Cinbis M, Ayter S, Anlar B, Aysun S. Familial segmental neurofibromatosis. I Child Neurol. 2004; 19:392-4. 